\title{
Acknowledgment to Reviewers of Condensed Matter in 2021
}

\author{
Condensed Matter Editorial Office
}

MDPI AG, St. Alban-Anlage 66, 4052 Basel, Switzerland

Citation: Condensed Matter Editorial

Office. Acknowledgment to

Reviewers of Condensed Matter in

2021. Condens. Matter 2022, 7, 14.

https://doi.org/10.3390/

condmat7010014

Published: 25 January 2022

Publisher's Note: MDPI stays neutral with regard to jurisdictional claims in published maps and institutional affiliations.

Copyright: (c) 2022 by the author. Licensee MDPI, Basel, Switzerland. This article is an open access article distributed under the terms and conditions of the Creative Commons Attribution (CC BY) license (https://creativecommons.org/license s/by/4.0/).

Rigorous peer-reviews are the basis of high-quality academic publishing. Thanks to the great efforts of our reviewers, Condensed Matter was able to maintain its standards for the high quality of its published papers. Thanks to the contribution of our reviewers, in 2021, the median time to first decision was 17 days and the median time to publication was 44 days. The editors would like to extend their gratitude and recognition to the following reviewers for their precious time and dedication, regardless of whether the papers they reviewed were finally published:

Abbrescia, Marcello

Abednatanzi, Sara

Adhikari, Rajdeep

Agosta, Charles

Anagnostopoulos, Dimitris

Arras, Rémi

Awoga, Oladunjoye A.

Baker, Peter

Baranovskii, Sergei

Barbiellini, Bernardo

Bellucci, Stefano

Bianconi, Antonio

Bjeliš, Aleksa

Bomantara, Radity

Breznay, Nicholas

Bruno, Flavio Y.

Camacho-Guardian, Arturo

Campi, Gaetano

Cappelluti, Emmanuele

Carter, W. Craig

Chen, Lung-Chien

Cheskis, Dima

Cho, Myung Hoon

Citro, Roberta

Da Silva Neto, Marcello Barbosa

Das, Mukunda

De Mello, Evandro Vidor Lins

Del Ben, Mauro

Di Castro, Carlo

Diamantini, Maria Cristina

Diep, Hung T.

Efremov, Dmitri

Elder, Ken

Escrig, Juan
Figueiras, Fábio Gabriel

Filipescu, Mihaela

Fujita, Masaki

Garbarino, Gaston

García-March, Miguel Ángel

Glenneberg, Jens

Gloter, Alexandre

Golubov, Alexander A.

Grilli, Marco

Grüner, Florian

Hönnicke, Marcelo Gonçalves

$\mathrm{Hu}$, Lun-Hui

Iatsunskyi, Igor

Ishikawa, Tetsuya

Karydas, Andreas

Kasamatsu, Kenichi

Khishchenko, Konstantin V.

Kichanov, Sergey

Kita, Takafumi

Kitamoto, Shunjii

Kitazawa, Takafumi

Koblischka, Michael R.

Konsolakis, Michalis

Kremer, Reinhard

Krivovichev, Sergey V.

Kř́žžek, Filip

Kuroki, Kazuhiko

Lakhno, Victor Dmitrievich

Lan, Si

Létoublon, Antoine

Liu, Tong

Lorenzana, José

Manzhos, Sergei

Mardegan, Jose R. L. 
Melnikov, Alexander S.

Micnas, Roman

Moskvin, Alexander S.

Muscas, Giuseppe

Nabiałek, Marcin

Nag, Abhishek

Nagasaka, Masanari

Nakagawa, Takahide

Nickles, Jerry

Nielsen, Stefan Kragh

Noce, Canio

Ohtsu, Hiroyoshi

Pagot, Gioele

Paluch, Andrew S.

Pan, Shilie

Pašti, Igor A.

Pereira, Luiz

Pini, Davide

Prabhakaran, Dharmalingam

Proux, Olivier

Pshenay-Severin, Dmitry A.

$\mathrm{Pu}$, Shengli

Raffy, Helene

Ramazanov, Shikhgasan

Refaely-Abramson, Sivan

Reguera, Edilso

Rodriguez, Aurelio Hierro

Ruys, Andrew

Rzazewski, Kazimierz

Sakai, Masamichi
Santos, Andrés

Sasaki, Nobuo

Sassi, Michel

Sedrakian, Armen

Seibold, Goetz

Seidlmayer, Stefan

Sgroi, Mauro Francesco

Shanenko, Arkady

Shimazoe, Kenji

Shirai, Kouun

Sidorenko, Anatolie

Sosorev, Andrey Y.

Spizzo, Federico

Talantsev, Evgeny F.

Tamburini, Fabrizio

Termine, Roberto

Thudiyangal, Mithun

Tinti, Gemma

Tocchio, Luca

Trifunovic, Luka

Trioni, Mario Italo

Tschauner, Oliver

Vasco, Edwin Herrera

Verucchi, Roberto

Virgilli, Enrico

Volosniev, Artem

Wang, Chun-Kai

Webb, Michael A.

Wysokinski, Karol Izydor

Zegrodnik, Michal 